\title{
Syrup Dosage Form Category
}

National Cancer Institute

\section{Source}

National Cancer Institute. Syrup Dosage Form Category. NCI Thesaurus. Code C154604.

A type of liquid pharmaceutical dose form consisting of a viscous liquid usually with a high concentration of sugar or other sweetening agent. 